\title{
A dynamic adjustable contention period mechanism and adaptive backoff process to improve the performance for multichannel mesh deterministic access in wireless mesh LAN
}

\author{
Hung-Chi Chien ${ }^{1}$, Bih-Hwang Lee ${ }^{1 *}$, Huai-Kuei Wu${ }^{2}$, Wen-Pin Hsu ${ }^{1}$ and Hsin-Hung Hsieh ${ }^{1}$
}

\begin{abstract}
IEEE802.11s draft proposes a new medium access control function-mesh deterministic access (MDA), which is mainly used for single-channel wireless mesh local area network (LAN). In single-channel environment, collisions between control packets and data packets may occur very often. To completely avoid the collision between control packets and data packets, the mesh delivery traffic indication message (DTIM) interval is first divided into contention period and data transmission period. To reduce the hardware requirements in design, we require a mesh point (MP) only equips a single transceiver to support multichannel environment. To provide higher performance and network capacity than the original MDA for wireless mesh LAN, we proposed a multichannel MDA (MMDA) algorithm. However, the MMDA algorithm may suffer from the resource waste problem when wireless mesh LAN is at heavy-loading situation, so this paper proposes a dynamic adjustable contention period (DACP) mechanism to solve this problem. In addition, we use an adaptive backoff process (ABP) to improve the fairness of the MMDA algorithm. The theoretical analysis gives the upper limit of the throughput for the DACP mechanism. The simulation experiments clearly show the results in multichannel wireless mesh LAN environment that the proposed scheme performs better than the MMDA algorithm and the enhanced distributed channel access (EDCA) in throughput, average waiting time, and packet drop ratio.
\end{abstract}

Keywords: IEEE802.11s; Medium access control; Mesh deterministic access; Wireless mesh LAN

\section{Introduction}

With the popularity of wireless local area network (LAN), increasing the network coverage is the necessary development. IEEE802.11s draft [1] divides nodes into two categories, including mesh node and non-mesh node. A mesh node can support mesh services, but non-mesh node cannot, e.g., the original IEEE802.11 station (STA) is a non-mesh node [2]. Mesh nodes are divided into two categories: mesh point (MP), which only supports mesh services such as route selection and packet forwarding; mesh access point (MAP), which provides access point (AP) functions for STAs and mesh services. For compatibility with the IEEE802.11 LAN, wireless mesh LAN must be able to link with other networks; therefore, a mesh

\footnotetext{
* Correspondence: bhlee@mail.ntust.edu.tw

${ }^{1}$ National Taiwan University of Science and Technology, 43, Section 4, Keelung Rd., Taipei 106, Taiwan

Full list of author information is available at the end of the article
}

point portal (MPP) provides the functions of layer 2 bridge and layer 3 interworking [3]. Wireless mesh LAN topology can be mainly divided into 802.11 connected mesh and 802.11 mesh ad hoc [4]. In IEEE802.11s draft, the medium access control (MAC) layer protocol has great influence for wireless mesh LAN performance $[5,6]$ and it follows the original IEEE802.11e enhanced distributed channel access (EDCA) [7], including congestion control $[8,9]$, power saving [10], synchronization [11] and beacon collision avoidance [12], and quality of service (QoS) [13-15].

IEEE802.11s draft proposes the mesh deterministic access (MDA) in the MAC enhanced region [16]. MDA mainly works in the single-channel network, and the related works of MDA also focuses on the improvement of single-channel network performance. The authors of [17] propose a semi-random backoff (SRB) method that enables resource reservation. Cheng et al. propose the DPSO-CA 
algorithm to assign channel with topology preservation in multiradio wireless mesh networks [18]. Xiao et al. investigate the fundamental performance limits of MAC protocols for particular multihop sensor networks [19]. Vishnevsky et al. make the beacons responsible for supporting MDA designed for delay-sensitive multimedia applications [20]. The authors of [21] develop a scheduled mesh access (SMA) mechanism based on MDA, while SMA has better anti-interference ability than EDCA. The authors of [22] define the MDA interframe space (MIFS) to make MP get higher priority to access its own MDA opportunity (MDAOP). However, single-channel network environment has higher collision probability and cannot avoid collisions between control packets and data packets. In particular, network performance is strictly limited in the single-channel wireless mesh LAN when it is in the heavy-loading situation and hidden node environment. According to $[23,24]$, using multichannel architecture is more effective than single-channel architecture to enhance performance and reduce collision probability in IEEE802.11 network. Nezhad et al. [25] shows some channel assignment (CA) to improve wireless mesh network performance by limiting the negative interference effects. The authors of [26] give the embedded version FreeBSD for multichannel wireless mesh networks. Ku et al. [27] proposes a traffic-aware switching scheme (TRASS) for wireless mesh network. In order to further enhance wireless mesh LAN performance and capacity, we have proposed multichannel MDA (MMDA) algorithm to make MDA works on multichannel wireless mesh LAN [28,29]. The MMDA algorithm adopts four-way handshaking mechanism to reduce hidden node problems, and mesh delivery traffic indication message (DTIM) interval is divided into contention period (CP) and data transmission period (DTP) to completely avoid the collision between control packets and data packets. The MMDA algorithm is designed with only single transceiver, so it can reduce the hardware requirements and design complexity.

The goals of this paper are to improve the MMDA algorithm and to enhance the performance of the multichannel wireless mesh LAN. The MMDA algorithm uses a fixed contention period mechanism, so it causes the network resource waste problem when wireless mesh LAN is in high-loading situation. This paper proposes a dynamic adjust contention period (DACP) mechanism to solve the abovementioned problem. Besides, the MMDA algorithm uses contention to reserve MDAOPs; hence, some MPs may reserve more MDAOPs than the other MPs, which cause unfairness. Therefore, this paper further adopts an adaptive backoff process (ABP) to improve fairness. The organization of this paper is as follows: the next section is background description; Section 3 is to describe the proposed schemes; Section 4 analyzes the throughput of the DACP mechanism; Section 5 shows the simulation results; and finally, Section 6 concludes the paper.

\section{Background description}

In the original MDA, a mesh DTIM interval is defined between two consecutive beacon frames. According to IEEE802.11s draft, the slot size of mesh DTIM interval is set to $32 \mu \mathrm{s}$, in which an MP can reserve MDAOP to transmit data. An MDAOP can be reused in every mesh DTIM interval until an MP finishes transmission and releases it. MDA is established by using two control packets: MDA setup request and MDA setup reply. Figure 1 shows the operation of MDA mechanism. Before a source $\mathrm{MP}\left(\mathrm{MP}_{\mathrm{A}}\right)$ transmits data, it sends MDA setup request packet to its destination MP for requesting MDAOP setup. If the destination $\mathrm{MP}\left(\mathrm{MP}_{\mathrm{B}}\right)$ successfully received MDA setup request packet and can satisfy the request of MDAOP setup, it will reply MDA setup reply packet to the source MP. Then, MDA mechanism is completed, so the source MP can build a MDAOP on selected location of mesh DTIM interval with the destination MP and periodically use this MDAOP to transmit data. However, MDA mechanism in IEEE802.11s draft is optional, and it means that not every MP in wireless mesh LAN should support MDA mechanism.

MMDA splits the mesh DTIM interval into CP and DTP as shown in Figure 2. In CP, MPs use contention mechanism to reserve MDAOP, and the successful MP establishes MDAOP at the selected channel to transmit data in DTP. It is to make sure that control packets and data packets can be transmitted in different intervals and avoid the collisions between them. Because MMDA algorithm allows an MP only equips a single transceiver in wireless mesh LAN, only a channel (i.e., $\mathrm{CH}_{1}$ ) is listened by all MPs in CP; then, all MPs know the channel

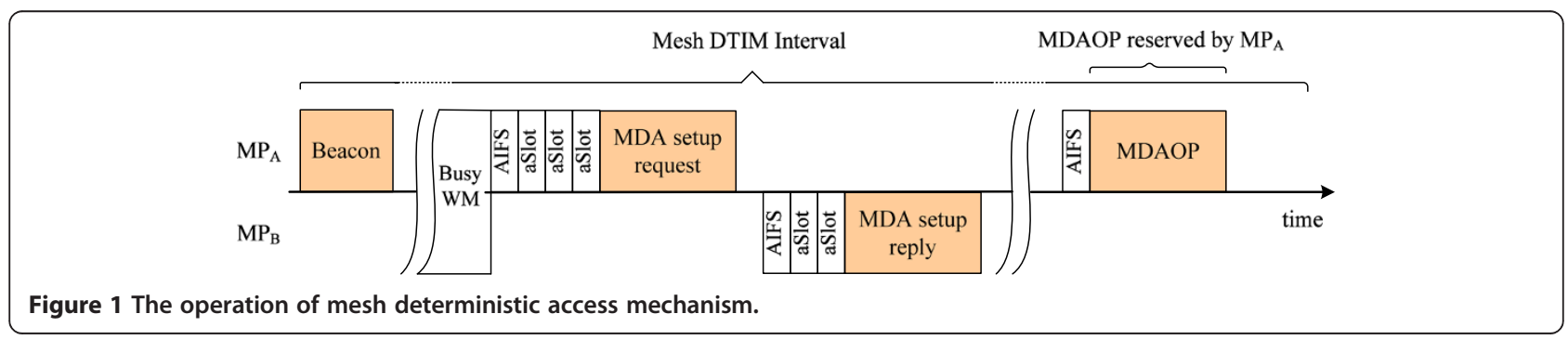




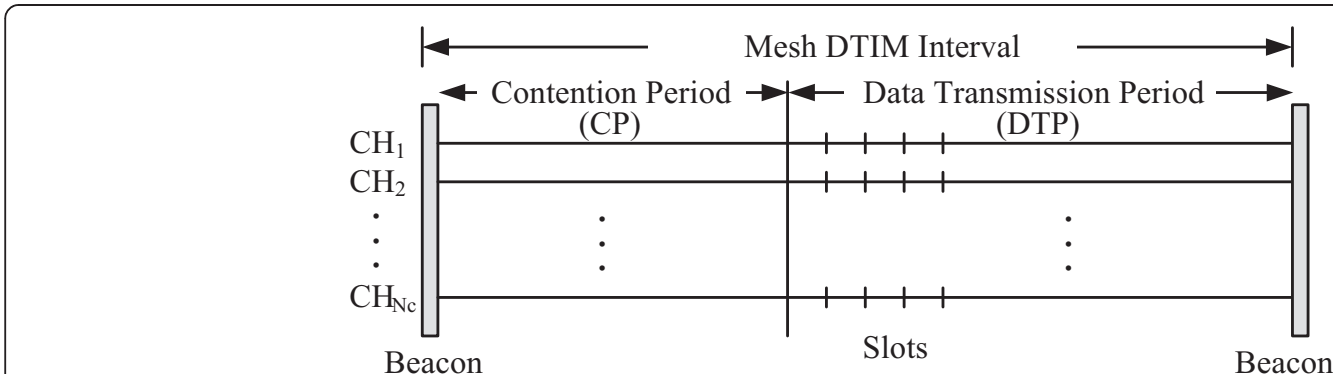

Figure 2 Mesh DTIM interval architecture of MMDA algorithm.

information in DTP, but $\mathrm{CH}_{1}$ can also be used to transmit data in DTP. The maximum size of an MDAOP to be reserved in DTP is 4,096 $\mu$ s (or 128 slots), which can be used by MP periodically at the same location of each DTP.

In order to make MPs achieve timing synchronization in wireless mesh LAN, we use the IEEE802.11 ad hoc timing synchronization mechanism [30] because the MMDA algorithm uses the new defined mesh DTIM interval architecture. An MP sends a beacon in accordance with the principles of the distributed coordination function (DCF) at the beginning of each beacon interval; the other MPs will cease sending beacons and take the timestamp values in the beacons if any MP sends beacon successfully. To use the MMDA algorithm, we require that each MP must build a neighbor MP status table (NMST) to make sure that each MP knows its neighbor MPs' status and channel state information, including the MP's identification (ID), channel ID currently used by the MP, offset from MDAOP's starting position to the beginning of subinterval, duration of MDAOP, periodicity (i.e., the number of MDAOPs in DTP), and MP supported for MDA mechanism or not. For example, if periodicity is four, it means that DTP is divided into four subintervals, while the four MDAOPs are located in the same distance from the beginning of these four subintervals.

Similarly as the original IEEE802.11 ad hoc networks, wireless mesh LAN also has hidden node problem [31]. In order to effectively solve this problem, the MMDA algorithm uses four-way handshaking mechanism instead of two-way handshaking mechanism in the original MDA. In other words, the MMDA algorithm uses four control packets, including MDA setup request, MDA setup reply, $M D A A C K$ (acknowledge), and $M D A A D V$ (advertisement), to reserve MDAOP. By the additional MDA ACK and MDA ADV, the MMDA algorithm can alleviate hidden node problem in wireless mesh LAN. The procedure of the MMDA four-way handshaking mechanism is shown in Figure 3. The proposed scheme in this paper also follows four-way handshaking mechanism of the MMDA algorithm.

The MMDA algorithm allows an MP to reserve different MDAOPs to transmit data on the same channel or different channels in DTP, if MP needs more than one MDAOP to meet the transmission request. Figure 4 shows an example of the multiple MDAOPs (included MDAOPs' offsets and durations) used by $\mathrm{MP}_{\mathrm{A}}$ on different channels $\left(\mathrm{CH}_{4}\right.$ and $\left.\mathrm{CH}_{5}\right)$ in DTP, and Table 1 shows that the NMST records the corresponding information of MDAOPs for this example.

The MMDA algorithm has two MDAOP selection mechanisms: the channel load first random fit (CLFRF) and the multichannel best fit (MCBF) mechanisms. The CLFRF mechanism is to randomly select duration from the lightest loading channel for MDAOP reservation, which has the advantage of simple and no more complex operation. The MCBF mechanism is to find the best fit location from all channels to build the requested MDAOP, i.e., the MCBF mechanism is to select the minimum duration from these durations; hence, the advantage of the MCBF mechanism is that the remaining space can be minimized after building MDAOP to avoid causing a large fragmentation.

\section{Dynamic adjustable contention period mechanism and adaptive backoff process}

The MMDA algorithm uses the fixed ratio of CP and DTP in the mesh DTIM interval, which enables MP to

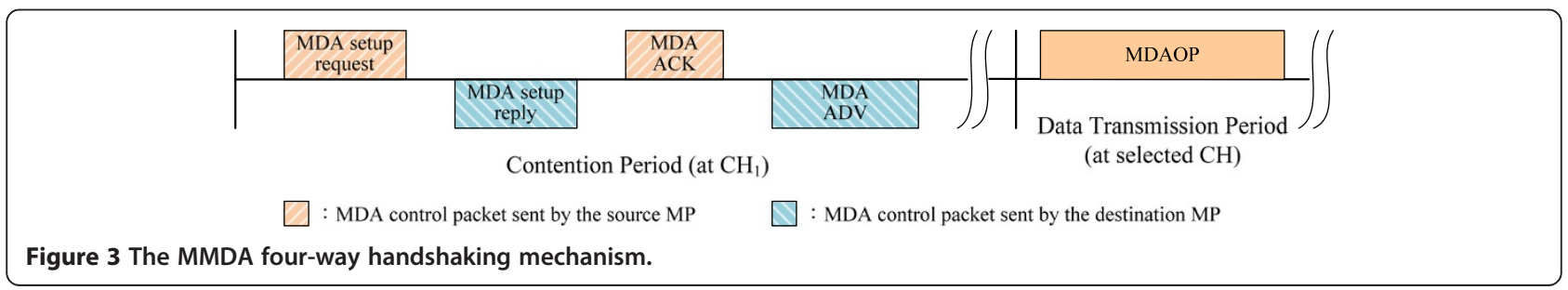




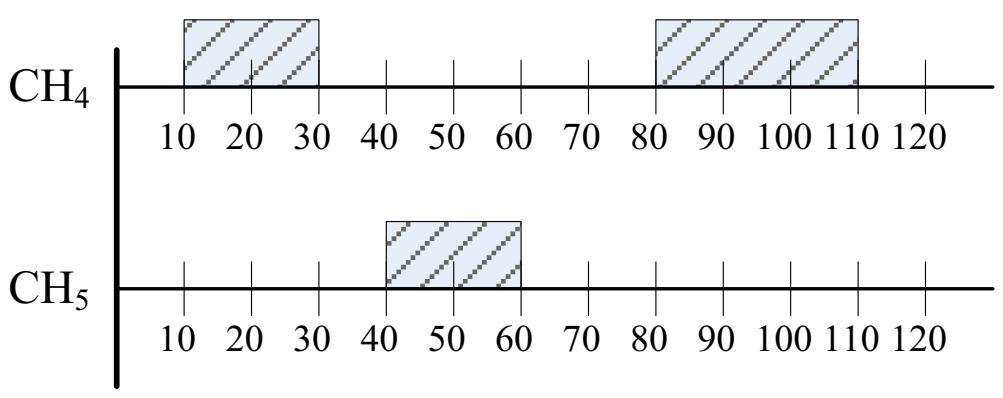

\section{: MPAOPs used by $\mathrm{MP}_{\mathrm{A}}$}

Figure 4 The example of the multiple MDAOPs used by $\mathrm{MP}_{\mathrm{A}}$ on different channels in DTP.

efficiently reserve enough MDAOPs in CP to transmit data. The available duration to establish MDAOPs in DTP will be almost reserved, if a wireless mesh LAN becomes heavily loaded. In addition, the holding time of the reserved MDAOP will increase, if the MDAOP owner needs to send a large amount of data by its reserved MDAOP periodically; therefore, the teardown ratio of MDAOPs in the wireless mesh LAN becomes seriously small, where teardown means MP must release the occupied MDAOP when it completes the transmitting data. An MP cannot find the available duration in DTP to establish MDAOP because each channel in DTP may be almost unable to provide the available duration. In this situation, MP will not send MDA setup request in $\mathrm{CP}$, and the times by using four-way handshaking mechanism to reserve MDAOP will be significantly reduced in $\mathrm{CP}$. The wasting resource problem may occur and cause the performance unable to further increase for wireless mesh LAN. In Figure 5, the shaded region in CP shows the wasted network resource of the MMDA algorithm. Therefore, the DACP mechanism and ABP are to solve for it and described as follows:

\subsection{Dynamic adjustable contention period mechanism}

The advantage of DACP mechanism is that it can further improve the capacity and performance of wireless mesh LAN in a heavy-loading situation. DACP mechanism allows MP to adjust CP ratio and increase DTP ratio in the mesh DTIM interval for sending more data when DTP is at the saturated condition. First, we define that MP may have three different MP types: normal contention

Table 1 The NMST record of the corresponding information of MDAOPs in Figure 4

\begin{tabular}{cccccc}
\hline MP ID & Channel ID & Offset & Duration & Periodicity & MDA support \\
\hline$M_{A}$ & 4 & 10 & 20 & 1 & 1 \\
$M_{A}$ & 5 & 40 & 20 & 1 & 1 \\
$M_{A}$ & 4 & 80 & 30 & 1 & 1 \\
\hline
\end{tabular}

period (NCP), enable contention period shorten (ECPS), and demarcation point (DP). NCP means that an MP maintains the original $\mathrm{CP}$ and DTP ratios in the mesh DTIM interval, while ECPS means that an MP shortens $\mathrm{CP}$ to increase the available duration in DTP because DTP has been saturated. The NCP MPs and ECPS MPs may cause the collisions between control packets and data packets when they communicate with each other because of different CP ratios. A DP is defined for the intermediate between NCP and ECPS, which means that the neighbor MPs of an ECPS MP should be the ECPS or DP type, and the neighbor MPs of an NCP MP should be the NCP or DP type. The duration in DP includes the shortened CP, the original DTP, and the additional period. Therefore, the DP MP has the following restrictions: (1) the DP MP in additional period can only receive control messages issued by its neighboring MPs to update NMST, and (2) the NCP or ECPS MP cannot reserve MDAOPs with a DP MP in additional period. The architectures of the mesh DTIM interval for these three types are different as shown in Figure 6, while Table 2 shows the three MP types' ratios of CP and DTP, where we assume that $X$ and $Y$ represent the original $\mathrm{CP}$ ratio of MMDA algorithm and the shortened CP ratio in the mesh DTIM interval. DACP mechanism allows MP to transfer among these three types according to its own situation and adjust the $\mathrm{CP}$ ratio of the mesh DTIM interval. The available duration which DTP gets from the shortened CP is called additional period. For example, the ECPS MP's DTP is the sum of the original DTP and the additional period.

In general, an MP uses the four-way handshaking mechanism of MMDA algorithm to reserve MDAOPs. An MP may determine to start the DACP mechanism as the network becomes heavy loading, i.e., if the utilization of DTP reaches almost saturated, and this situation remains over a certain duration (e.g., ten mesh DTIM intervals). Then, the MP sends a contention period change (CPC) request packet to its neighbor MPs and waits for the neighbor MPs' CPC reply packets to do the state 


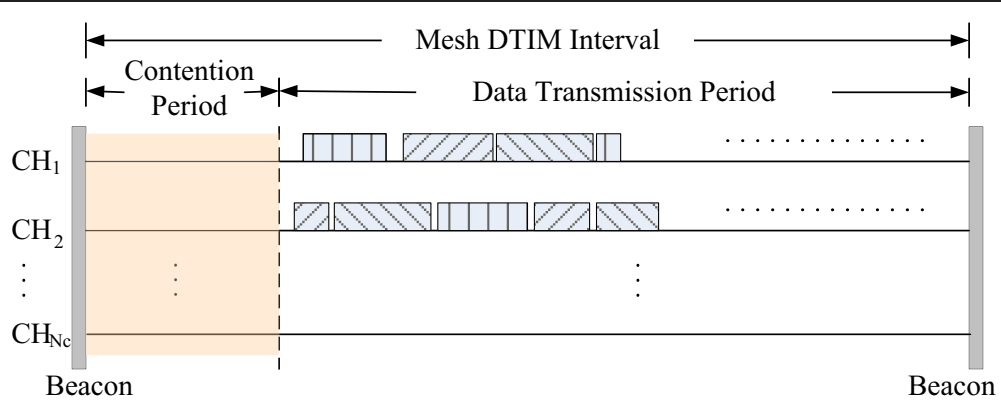

$\triangle$ : MDAOPs used by $\mathrm{MP}_{\mathrm{A}} \square:$ MDAOPs used by $\mathrm{MP}_{\mathrm{B}} \square:$ MDAOPs used by MP

Figure 5 Network resource waste problem of MMDA Algorithm.

transition. The related state transition diagram for these three different types is shown in Figure 7 with the actions marked from A to $\mathrm{K}$, where pre-NCP, pre-DP, and preECPS represent the three transient states before MP entering the NCP, DP, and ECPS states, respectively. The functions of the actions are summarized as the following:

A. The NCP MP records the information and replies the $\mathrm{CPC}$ reply packet when it receives the $\mathrm{CPC}$ request packet of NCP or DP type.
B. The NCP MP enters pre-ECPS state when the saturated situation occurs. It continues to send the $\mathrm{CPC}$ request packet of ECPS type until receiving all neighbor MPs' CPC reply packets.

C. The pre-ECPS MP enters the ECPS state in the next mesh DTIM interval, when it receives all neighbor MPs' CPC reply packets.

D. The NCP MP replies the CPC reply packet and enters the pre-DP state, when it receives the CPC request packet of ECPS. The pre-DP MP continues
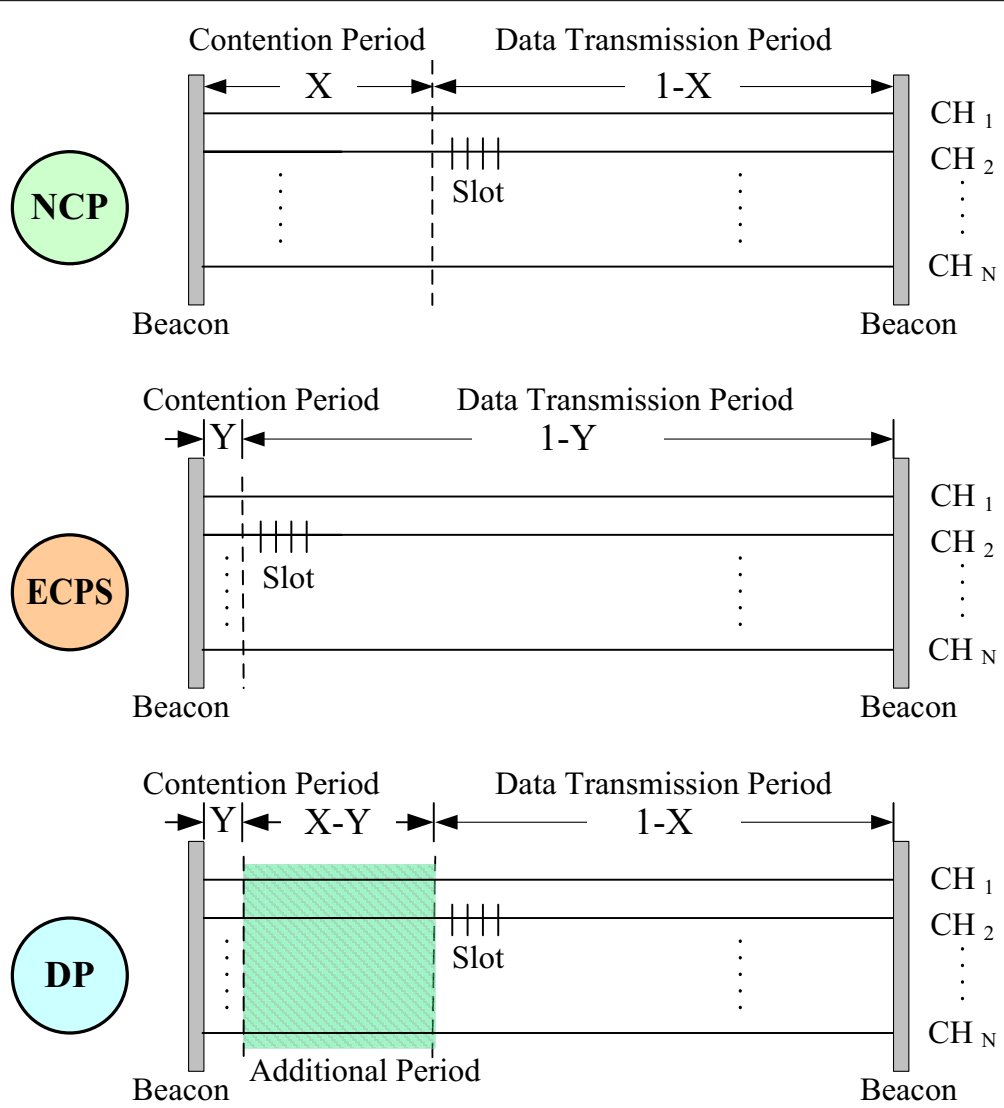

Figure 6 Mesh DTIM interval artictecture of the three different MP types. 
Table 2 The ratios of CP and DTP in the different MP types

\begin{tabular}{ll}
\hline Type & Mesh DTIM interval architecture \\
\hline NCP & CP ratio $: X$, DTP ratio $: 1-X$ \\
ECPS & CP ratio $: Y$, DTP ratio $: 1-Y$ \\
DP & CP ratio $: Y$, additional period ratio $: X-Y$, DTP ratio $: 1-X$ \\
\hline
\end{tabular}

to send $\mathrm{CPC}$ request packet of DP type until receiving all neighbor MPs' $\mathrm{CPC}$ reply packets.

E. The pre-DP MP enters the DP state in the next mesh DTIM interval, when it receives all neighbor MPs' CPC reply packets.

F. The ECPS MP records the information and replies the $\mathrm{CPC}$ reply packet, when it receives the CPC request packet of ECPS or DP type.

G. The ECPS MP enters the pre-DP state, when the utilization of DTP is less than the high-loading situation for more than the limit number of mesh DTIM intervals, and there is no MDAOP in the additional period. It continues to send the CPC request packet of DP type until receiving all neighbor MPs' CPC reply packets.

$\mathrm{H}$. The DP MP enters the pre-ECPS state, when the saturated situation occurs. It continues to send the $\mathrm{CPC}$ request packet of ECPS type until receiving all neighbor MPs' CPC reply packets.

I. The DP MP enters the pre-NCP state, when all DP MP's neighbor MPs are not ECPS type and the utilization of DTP is less than the high-loading situation for more than the limit number of mesh DTIM intervals. It continues to send the $\mathrm{CPC}$ request packet of NCP type until receiving all neighbor MPs' CPC reply packets.

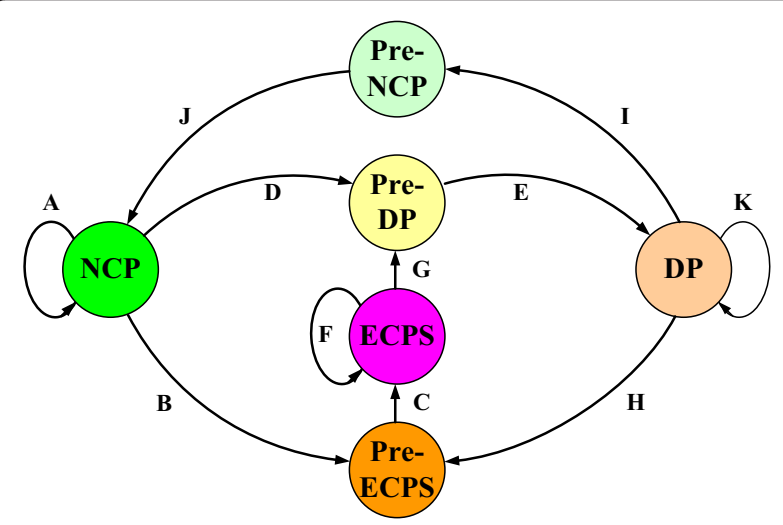

Figure 7 The related state transition diagram for the three different MP types.
J. The pre-NCP MP enters the NCP state in the next mesh DTIM interval, when it receives all neighbor MPs' CPC reply packets.

$\mathrm{K}$. The DP MP records the information and replies the $\mathrm{CPC}$ reply packet, when it receives any type of $\mathrm{CPC}$ request packet.

To further explain the operations of the DACP mechanism, we describe the state transition diagrams as shown in Figure 7 for the MP at the states of NCP, ECPS, and DP, respectively. First, we assume that the MP starts at the NCP state, it may stay at the NCP state by action A; it may go to the pre-ECPS state by action $\mathrm{B}$ and then arrive at the ECPS state by action $C$; or it may go to the pre-DP state by action $\mathrm{D}$ and then arrive at the DP state by action E. Then, we assume that the MP starts at the ECPS state, it may stay at the ECPS state by action F; or it may go to the pre-DP state by action $\mathrm{G}$ and then arrive at the DP state by action E. Similarly, we assume that the MP starts at the DP state, it may stay at the DP state by action K; it may go to the pre-ECPS state by action $\mathrm{H}$ and then arrive at the ECPS state by action C; or it may go to the pre-NCP state by action I and then arrive at the NCP state by action $\mathrm{J}$.

\subsection{Adaptive backoff process}

Before an MP starts contention to reserve MDAOP in CP, it first enters backoff procedure according to CSMA/CA in IEEE802.11 standard. After waiting for a random time, the source MP sends MDA setup request packet to the target MP. Because the MMDA algorithm uses the contention mechanism to reserve MDAOPs, an MP may reserve more MDAOPs than the other MPs and result in unfairness problem. The contention window (CW) size may play an essential role in improving fairness [32]; therefore, $\mathrm{ABP}$ is to adjust the backoff time parameters according to the number of MDAOPs owned by an MP, which allows the MP having less MDAOPs may have higher probability to reserve MDAOPs.

According to NMST, an MP knows the neighbor MPs' MDAOPs and decides to change the backoff time or not by comparing its own MDAOPs' duration and the average durations of the neighbor MPs' MDAOPs. In other words, ABP uses the evaluation parameter $r$ to decide if the MP needs to adjust the backoff time parameters or not as shown in (1), where MDAOP ${ }_{\text {own }}$ and MDAOP $_{\text {avg }}$ represent the durations of MDAOPs reserved by the MP itself and the neighbor MPs, respectively. According to the value $r$, ABP defines four access categories (AC), $\mathrm{AC}_{0} \sim \mathrm{AC}_{3}$, with different arbitration interframe space number (AIFSN), CW $\mathrm{CW}_{\min }$, and $\mathrm{CW}_{\max }$ as shown in Table 3. Then, the MP can get the corresponding backoff time parameters to derive the new backoff time by (2). 
Table 3 The backoff time parameters of ABP

\begin{tabular}{lcccc}
\hline AC & $r$ value & AIFSN & $\mathbf{C W}_{\text {min }}$ & $\mathbf{C W}_{\text {max }}$ \\
\hline $\mathrm{AC}_{0}$ & $r=0$ & 2 & 7 & 31 \\
$\mathrm{AC}_{1}$ & $0<r<0.5$ & 2 & 15 & 63 \\
$\mathrm{AC}_{2}$ & $0.5 \leqq r<1$ & 2 & 31 & 127 \\
$\mathrm{AC}_{3}$ & $r \geqq 1$ & 2 & 63 & 255 \\
\hline
\end{tabular}

The advantage of ABP is that it can enhance fairness between MPs in wireless mesh LAN.

$$
r=\frac{\text { MDAOP }_{\mathrm{own}}}{\mathrm{MDAOP}_{\mathrm{avg}}}
$$

$$
\text { Backoff_Time }[\mathrm{AC}]=\operatorname{Random}(0, \mathrm{CW}[\mathrm{AC}]) \times \text { aSlotTime }
$$

\section{Analysis of DACP mechanism in throughput}

In this section, we analyze the MMDA algorithm with the DACP mechanism based on the saturated contention in CP. We assume that there are $n$ MPs operated in CP with the saturation mode; we only consider the two-hop wireless mesh LAN environment to simplify the analysis. We assume that there are totally $n$ MPs operated in CP with the saturation mode binary exponential backoff (BEB). Let us denote $W_{0}$ be the initial contention window, while $p_{t}$ and $p_{c}$ are the probabilities of the MP transmission and the collisions caused by at least one of $n-1 \mathrm{MP}$ transmissions, respectively, which can be obtained by (3) [33], where MPs contend with each other until successful transmission in $\mathrm{CP}$ without limiting the maximum backoff stage. $p_{t}$ can also be obtained by (4).

$$
\begin{aligned}
& p_{t}=\frac{2\left(1-2 p_{c}\right)}{W_{0}\left(1-p_{c}\right)+1-2 p_{c}} \\
& p_{t}=1-\left(1-p_{c}\right)^{1 /(n-1)}
\end{aligned}
$$

We can easily obtain the unique solutions of $p_{t}$ and $p_{c}$, respectively, by solving (3) and (4), if $n$ and $W_{0}$ are given. In addition, $p_{t}(n)$ and $p_{c}(n)$ can be considered as the functions of $n$ for $p_{t}$ and $p_{c}$, respectively, by assuming $W_{0}$ is a fixed system parameter. Therefore, the probabilities of successful transmission and idle channel can also be considered as a function of $n$ and expressed by (5) and (6), respectively. Let $p_{\text {coll }}$ represent the collision probability caused by two or more MP transmissions at the same time, and $p_{\text {coll }}(n)$ is the function of $n$ for $p_{\text {coll }}$ as shown in (7). Similarly, we consider the other parameters as the functions of $n$ later in this paper.

$$
p_{\text {succ }}(n)=n p_{t}(n)\left(1-p_{t}(n)\right)^{n-1}
$$

$$
\begin{aligned}
& p_{i}(n)=\left(1-p_{t}(n)\right)^{n} \\
& p_{\text {coll }}(n)=1-p_{i}(n)-p_{\text {succ }}(n)
\end{aligned}
$$

According to the content of NMST, MP knows the durations will be used in DTP and avoids selecting these durations; then, MP can reserve MDAOP by completing the MMDA four-way handshaking mechanism. Let us denote $T_{\mathrm{cp}}$ to be the duration of CP, which obviously affects the number of successful contentions in CP. Let us also denote $T_{\mathrm{cp} \_ \text {c }}$ to be the critical duration of $\mathrm{CP}$, which is the minimum duration of $\mathrm{CP}$ for MPs to successfully reserve all available MDAOPs. Let us assume that an MP will experience $i$ times of collision and $j$ empty slot times before successfully reserving an MDAOP in CP with $n$ MPs to contend, whose joint probability mass function, $P_{\mathrm{ss}}(i, j, n)$, can be obtained by (8), where $I$ and $J$ are the random variables that an MP will experience $i$ times of collision and $j$ empty slots before successfully reserving an MDAOP in $\mathrm{CP}$, respectively. Furthermore, the means of $I$ and $J$ can be expressed as the functions of $n$ and obtained by (9) and (10), respectively.

$$
\begin{aligned}
& p_{s s}(i, j, n)=p[I=i, J=j] \\
&=\left(\begin{array}{l}
i+j \\
j
\end{array}\right) p_{\text {coll }}^{i}(n) p_{i}^{j}(n) p_{\text {succ }}(n), \text { for } i \\
&=0,1,2 \ldots \text { and } j=0,1,2 \ldots \\
& \bar{I}(n)=\sum_{i=0}^{\infty} i \times \sum_{j=0}^{\infty} p_{\mathrm{ss}}(i, j, n) \\
& \bar{J}(n)=\sum_{j=0}^{\infty} j \times \sum_{i=0}^{\infty} p_{\mathrm{ss}}(i, j, n)
\end{aligned}
$$

Let us denote $T_{s}$ and $T_{c}$ to be the durations of each successful MMDA four-way handshaking contention and collision as shown in (11) and (12), respectively, where $T_{\text {MDASRQ }}, T_{\text {MDASRB }}, T_{\text {MDAACK}}, T_{\text {MDAADV}}, T_{\text {DIFS }}$, and $T_{\text {SIFS }}$ are the durations of MDA setup request, MDA setup reply, MDA acknowledge, MDA advertisement, DIFS, and SIFS, respectively. Let us define $T_{r}(n)$ as the average time to reserve an MDAOP for an MP in CP, which can be obtained by (13), where $T_{i}$ is a slot time that should be long enough to access the channel state information. Let us denote $T_{\text {DTIM }}$ and $T_{\text {DTP }}$ to be the durations of the mesh DTIM interval and data transmission period, respectively, where $T_{\mathrm{DTP}}$ can be obtained by (14).

$$
\begin{aligned}
& T_{s}= T_{\mathrm{MDASRQ}}+T_{\mathrm{MDASRP}}+T_{\mathrm{MDAACK}} \\
&+T_{\mathrm{MDAADV}}+T_{\mathrm{DIFS}}+3 T_{\mathrm{SIFS}} \\
& T_{c}= T_{\mathrm{MDASRQ}}+T_{\mathrm{DIFS}} \\
& T_{r}(n)=\bar{I}(n) \times T_{c}+\bar{J}(n) \times T_{i}+T_{s}
\end{aligned}
$$




$$
T_{\mathrm{DTP}}=T_{\mathrm{DTIM}}-T_{\mathrm{cP}}
$$

Let us denote $N_{a}$ to be the available MDAOPs provided for MPs to reserve in DTP as shown in (15), where $N_{c}, B, D$, and $L$ are the number of channels in wireless mesh LAN, each channel's bandwidth, MDAOP duration, and the average interval between two MDAOPs in DTP, respectively. Let us define $N_{\min }$ as the minimum value of $n$ and $N_{a}$; then, the critical duration of CP $T_{\mathrm{cp}_{-} \mathrm{c}}$ (n) can be obtained by (16). Hence, the available MDAOPs will be completely reserved by MPs within $\mathrm{CP}$, if $T_{\mathrm{cp}}$ is longer than $T_{\mathrm{cp}}{ }_{\mathrm{c}}$. Let us define $T_{m}$ as the $m$ th subcritical duration of CP that $m$ MPs can successfully reserve MDAOPs, which can be obtained by (17). We define $G(n)$ as the number of MPs that can successfully reserve MDAOPs in a CP, which can be obtained by (18). Finally, the average throughput of the NCP MP, $S_{\mathrm{NCP}}(n)$, can be obtained by (19).

$$
\begin{aligned}
& N_{a}=\left[\frac{T_{\mathrm{DTP}} \times B}{D+L}\right] \times N_{c} \\
& T_{\mathrm{cp} \_\mathrm{c}}(n)=\sum_{x=0}^{N_{\min }} T_{r}(n-x) \\
& T_{m}(n)=\sum_{x=0}^{m-1} T_{r}(n-x), \text { for } 1 \leq m \leq N_{\min } \\
& G(n)=\min (n, m) T_{\mathrm{cp}} \in\left[T_{m}, T_{m+1}\right), \text { for } \\
& m=0,1, \ldots, N_{\mathrm{min}-1} \\
& S_{\mathrm{NCP}}(n)=\frac{G(n) \times D}{T_{\mathrm{DTIM}}}
\end{aligned}
$$

Next we analyze the throughput for the ECPS MP. An MP will enter the ECPS state and shorten the CP of the mesh DTIM interval if DTP becomes saturated. Let us define $T_{\mathrm{cP}_{-} \mathrm{s}}$ and $T_{\mathrm{ap}}$ to be the shortened $\mathrm{CP}$ and the duration of the additional period, respectively. Let us also define $N_{\text {ap }}$ to be the available MDAOPs provided for MPs to reserve in the additional period, which can be obtained by (20). The shortened CP ratio $T_{\mathrm{cp}_{-} \mathrm{s}}(n)$ can be obtained by (21). Let denote $G_{s}(n)$ be the number of MPs that can successfully reserve MDAOPs in an additional period, if $T_{\mathrm{cp}_{-} \mathrm{s}}$ is long enough for MPs to reserve the available MDAOPs in additional period, which can be obtained by (22). Finally, the throughput of the ECPS MPs, $S_{\mathrm{ECPS}}(n)$, can be obtained by (23).

$$
\begin{aligned}
& N_{\text {ap }}=\left[\frac{T_{\text {ap }} \times B}{D+L}\right] \times N_{c} \\
& T_{\mathrm{cp}_{\mathrm{s}}}(n)=\sum_{x=0}^{N_{\text {ap }}} T_{r}(n-x)
\end{aligned}
$$

$$
\begin{aligned}
& G_{s}(n)=G(n)+N_{\mathrm{ap}} \\
& S_{\mathrm{ECPS}}(n)=\frac{G_{s}(n) \times D}{T_{\mathrm{DTIM}}}
\end{aligned}
$$

\section{Simulation experiments}

In this section, simulation has been executed by using C\# program. We consider the wireless mesh LAN topology in a random style, which consists of 32 MPs placed in $250 \times 250 \mathrm{~m}$ region. We assume that all MPs are fixed with the traffic type of constant bit rate (CBR), regardless of background noise and propagation delay. We also assume that the original $\mathrm{CP}$ ratio $(X)$ and the shortened $\mathrm{CP}$ ratio $(Y)$ are $30 \%$ and $5 \%$, respectively. The other simulation parameters are listed in Table 4.

The simulation experiments show for the performance in average throughput, average waiting time, packet drop ratio, fairness, and collision ratio, which are defined in (24) to (28), respectively. In (24), $\mathrm{Pkt}_{i}, s, T$, and $M$ represent the total number of the transmitted packets by $\mathrm{MP}_{i}$, packet size, total simulation time, and total number of MPs, respectively. In (25), Tx_Pkt, $\mathrm{Tl}_{i}$, and $\mathrm{Ta}_{i}$ represent the total success transmitted packets, the times that the $i$ th packet leaves and enters the queue, respectively. In (26), $\mathrm{Pkt}_{g}$ and $\mathrm{Pkt}_{d}$ represent the total numbers of generated and dropped packets, respectively. In (27), $S_{i}$ represents the throughput of $\mathrm{MP}_{i}$. In (28), $N_{\text {coll }, i}$ and $N_{\text {cont }, i}$ represent the total numbers of collisions and contentions occurred at $\mathrm{MP}_{i}$, respectively.

$$
\begin{aligned}
& \text { Average_throughput }=\frac{\sum_{i=1}^{n} \mathrm{Pkt}_{i} \times s}{n \times T} \\
& \text { Avg_waiting }_{\text {time }}=\frac{\sum_{i=1}^{\text {Tx_Pkt }}\left[\mathrm{Tl}_{i}-\mathrm{Ta}_{i}\right]}{\text { Tx_Pkt }}
\end{aligned}
$$

Table 4 The simulation parameters

\begin{tabular}{cc}
\hline Parameter & Value \\
\hline Number of MPs & 32 \\
Slot time & $32 \mathrm{\mu s}$ \\
Mesh DTIM interval & $100 \mathrm{~ms}$ \\
Packet size & 512 bytes \\
MP transmission range & $60 \mathrm{~m}$ \\
Number of channels & 3 \\
Channel capacity & $2 \mathrm{Mbps}$ \\
dot11MeshRetryTimeout & $500 \mathrm{~ms}$ \\
dot11MeshMaxRetries & 6 \\
Simulation time & $100 \mathrm{~s}$ \\
\hline
\end{tabular}




$$
\begin{aligned}
& \text { Packet_drop_ratio }=\frac{\mathrm{Pkt}_{d}}{\mathrm{Pkt}_{g}} \\
& \text { Fairness_index }=\frac{\left(\sum_{i=1}^{n} S_{i}\right)^{2}}{n \times \sum_{i=1}^{n}\left(S_{i}\right)^{2}} \\
& \text { Collision_ratio }=\frac{\sum_{i=1}^{n} N_{\text {coll }, i}}{\sum_{i=1}^{n} N_{\text {cont }, i}}
\end{aligned}
$$

Because the MAC layer protocol of IEEE802.11s draft follows EDCA, the performance of the proposed scheme is compared with EDCA and the MMDA algorithm in average throughput, average waiting time, packet drop ratio, fairness, and collision ratio in the simulation. There are three sets of results to be compared: the MMDA algorithm with DACP mechanism and ABP (called MMDA-DACP + ABP), EDCA using 5\% and $30 \%$ CP ratio (called EDCA-5\% and EDCA-30\%), and the MMDA algorithm using 5\% and 30\% CP ratio of the mesh DTIM interval (called MMDA-5\% and MMDA$30 \%)$, respectively, where the traffic load is varied from 0.1 to 1.0 (i.e., fully loaded at $375 \mathrm{kbps}$ for each MP).

From Figures 8, 9, 10, we find EDCA has the worst performance than the other three schemes, because the source MP and the destination MP have to coordinate a channel to be used, and the source MP must contend with the other MPs in CP. In addition, the source MP needs to reserve transmission opportunity (TXOP) by contention mechanism in DTP, which increases network overhead and more collisions. Furthermore, the TXOP cannot periodically be used in every mesh DTIM interval, so it increases the numbers of contentions and causes EDCA having worst performance. We also find that MMDA$\mathrm{DACP}+\mathrm{ABP}$ performs better than MMDA-5\% and MMDA-30\% in average throughput, average waiting time, and packet drop ratio when traffic load increases because MMDA-DACP + ABP allows for dynamically adjusting $C P$ ratio and increasing DTP ratio in the mesh DTIM interval to improve wireless mesh LAN performance. Because the CP ratio of MMDA$30 \%$ is always fixed at $30 \%$, this results in the network resources wasted and leads to the poor performance than MMDA-DACP + ABP and MMDA-5\% when wireless mesh LAN is at heavy-loading situation. MMDA-5\% has better performance than MMDA-30\% in heavy loading, but the shortened CP of MMDA-5\% is not enough for MPs to efficiently reserve MDAOPs at light loading. Therefore, in Figures 9 and 10, the average waiting time and the packet drop ratio of MMDA-5\% are greater than MMDA-DACP + ABP and MMDA-30\% at light loading.

Figure 11 shows the fairness comparison for these three schemes. We find that MMDA-DACP + ABP has better fairness than the MMDA algorithm because it uses adaptive backoff process and helps the MPs having less MDAOPs have higher probability to reserve MDAOPs. The adaptive backoff process also helps MMDA-DACP + ABP to reduce collision ratio as shown in Figure 12 because the adaptive backoff process divides

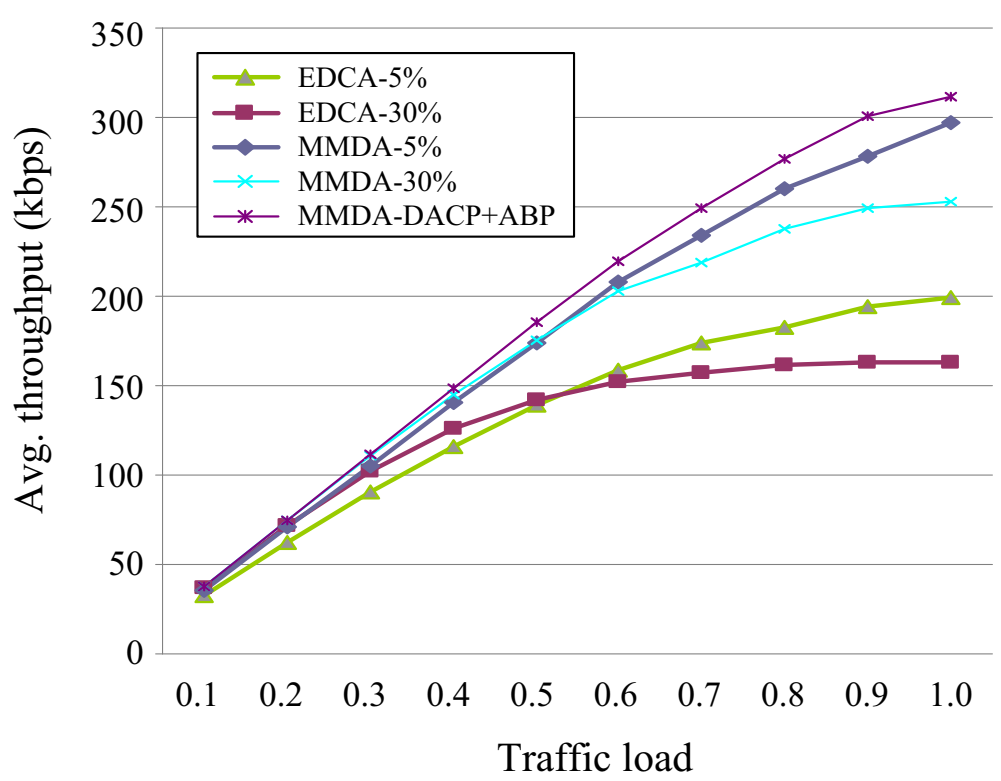

Figure 8 Average throughput versus traffic load for the three schemes. 


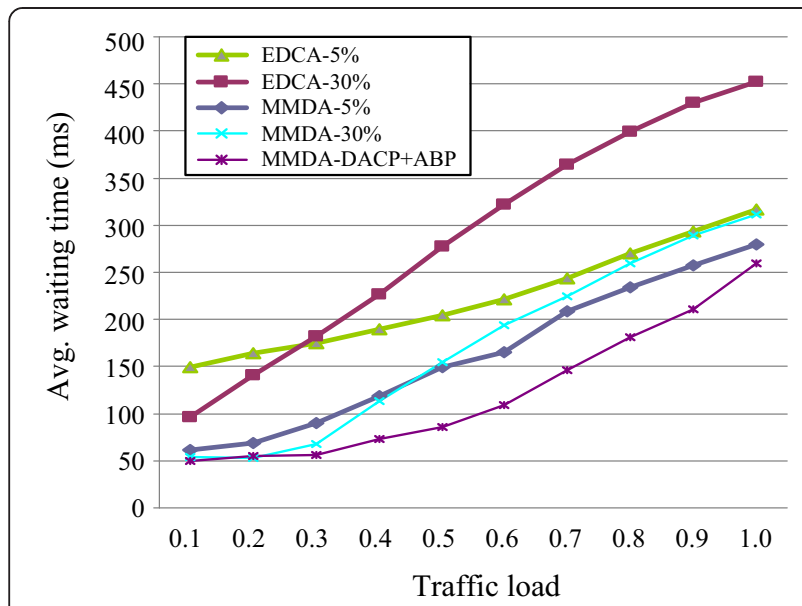

Figure 9 Average waiting time versus traffic load for the three schemes.

MPs into four categories with different backoff time parameters, respectively. EDCA has the worst collision ratio because it has to contend both in CP and DTP.

Figure 13 shows the average throughput changed by the three different MP types in MMDA-DACP + ABP. It is obvious that all MPs in wireless mesh LAN are NCP at low traffic load, and their throughput increases when traffic load increases. However, most of the NCP MPs will start DACP to change their states to ECPS because of DTP saturated when traffic load becomes heavier than $50 \%$. As traffic load becomes heavier, the NCP MP gradually transits to ECPS, i.e., the ECPS MPs' throughput increases and the NCP MPs' throughput decreases.

In order to observe how the MPs change for the three different types using the MMDA algorithm with DACP and ABP, we set the network traffic to be fully loaded (i.e., $375 \mathrm{kbps}$ for each MP) before $50 \mathrm{~s}$ of the simulated time and lightly loaded (i.e., 37.5 kbps for each MP) after

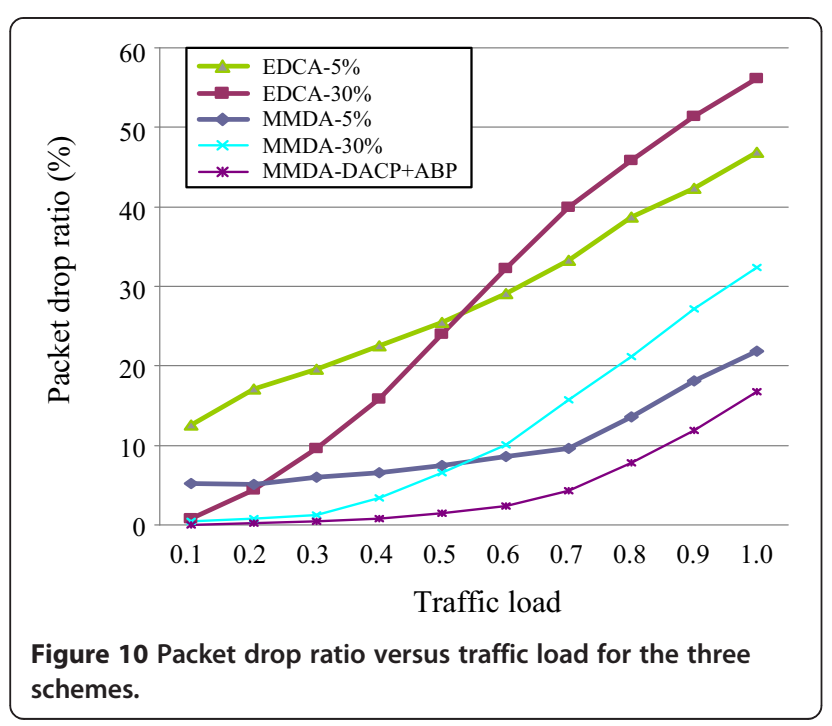

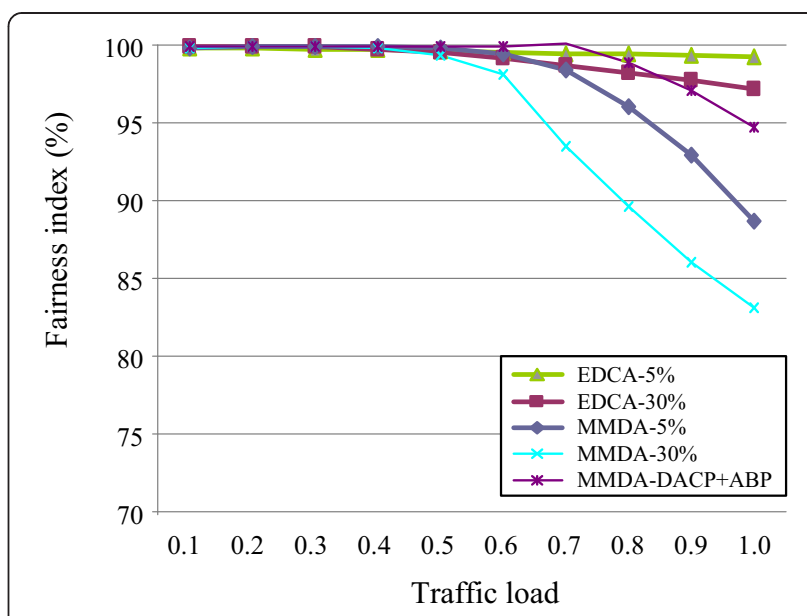

Figure 11 Fairness comparisons versus traffic load for the three schemes.

50 s. Figure 14 shows that the MPs start DACP to change type at the fourth second and completely transit from NCP to ECPS after $0.4 \mathrm{~s}$ at the fully loaded traffic, while the MPs completely transit to NCP at the $53.5 \mathrm{~s}$ of the simulated time at the lightly loaded traffic.

\section{Conclusions}

Multichannel MDA (MMDA) algorithm can make full use of multichannel environment to improve the overall performance and capacity of wireless mesh LAN, but the MMDA algorithm uses fixed contention period mechanism which causes the waste problem of network resources when wireless mesh LAN is at heavy-loading situation. Besides, the MMDA algorithm using the contention mechanism to reserve MDAOPs will cause the unfairness problem between MPs.

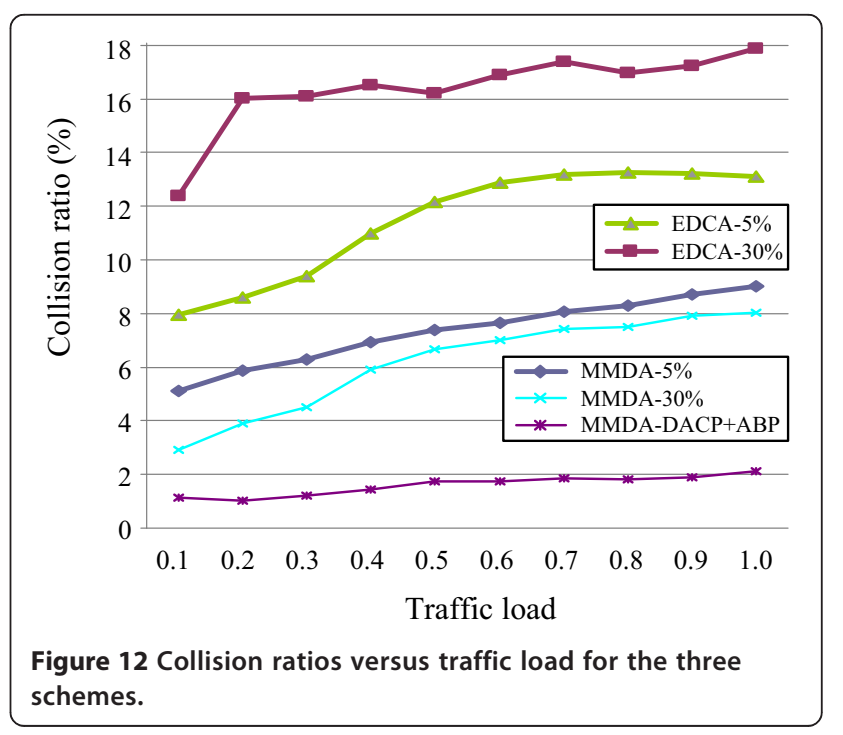




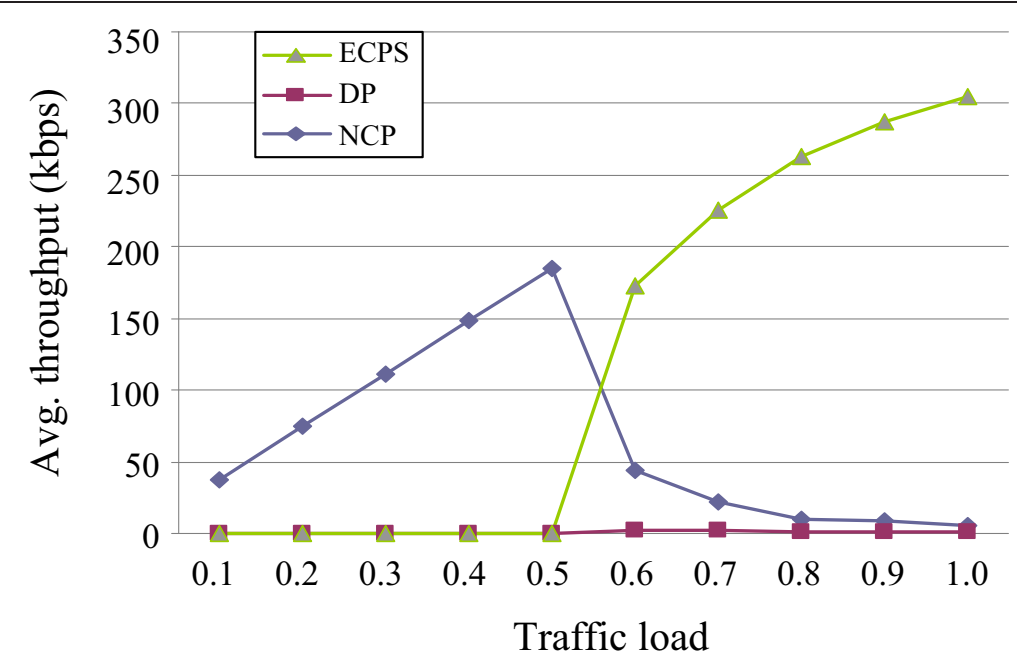

Figure 13 The average throughput versus traffic load for the three different MP types using the MMDA algorithm with DACP and ABP.

The proposed scheme uses the DACP mechanism and $\mathrm{ABP}$, so it can further improve the network performance when wireless mesh LAN is at heavyloading situation and alleviate the unfairness problem. The simulation results clearly show that in heavyloading wireless mesh LAN environment, the MMDA algorithm using DACP mechanism and ABP outperforms the MMDA algorithm in average throughput, average waiting time, packet drop ratio, collision ratio, and fairness. The proposed scheme can truly improve the performance of the MMDA algorithm.

\section{List of symbols}

$r$ The evaluation parameter for $\mathrm{ABP}$

$\mathrm{MDAOP}_{\text {own }}$ The durations of MDAOPs reserved by the MP itself

$\mathrm{MDAOP}_{\text {avg }}$ The durations of MDAOPs reserved by neighbor MPs

$n$ Total numbers of MPs in wireless mesh LAN

$W_{0}$ The initial contention window

$p_{c}$ Collision probability caused by at least one of $n-1$ MP transmissions

$p_{t}$ Transmission probability of each MP

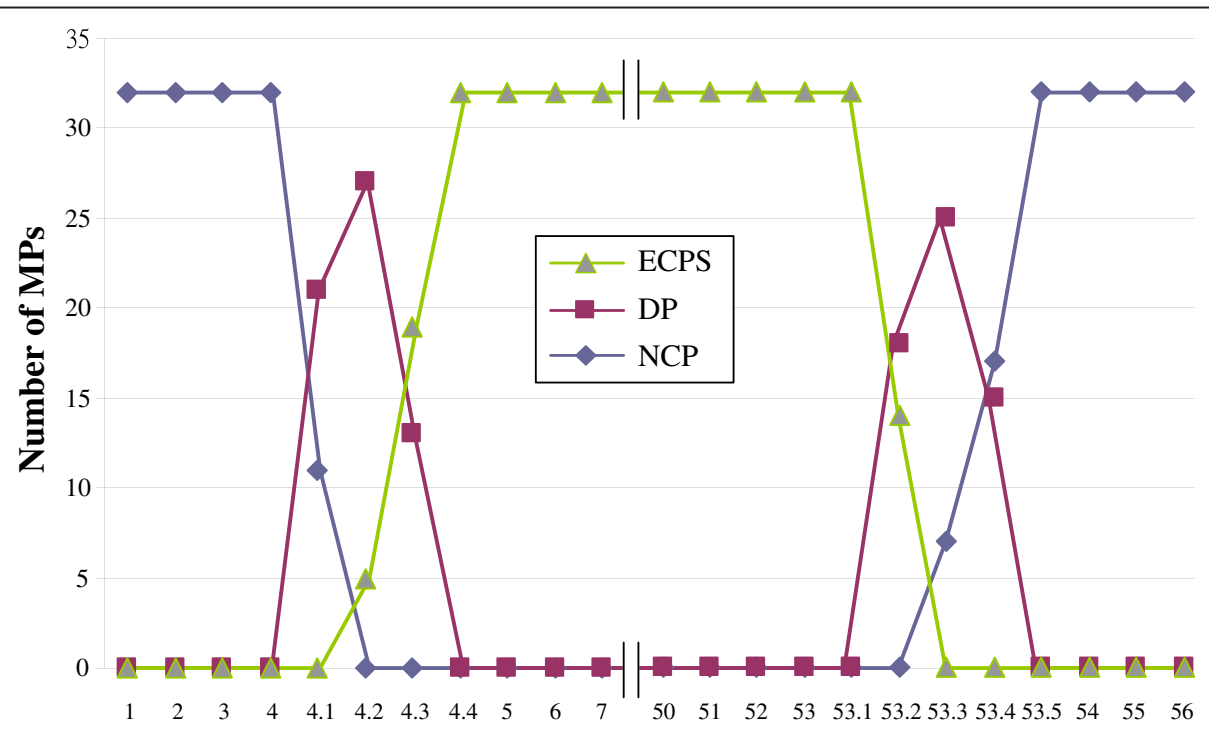

Simulation Time (seconds)

Figure 14 Number of nodes to change types versus simulation time for the three different MP types using the MMDA algorithm with $D A C P$ and $A B P$. 
$p_{\text {succ }}$ Probability of successful transmission

$p_{i}$ Probability of the idle channel

$p_{\text {coll }}$ Collision probability caused by two above MP transmissions

$T_{\mathrm{cp}}$ The durations of contention period

$T_{\text {cp_c }}$ The critical duration of $\mathrm{CP}$

$I$ A random variable; an MP will experience $i$ times of collision before successfully reserving a MDAOP in $\mathrm{CP}$

$J$ A random variable; an MP will experience $j$ empty slot times before successfully reserving a MDAOP in CP

$P_{\mathrm{ss}}(i, j, n)$ The joint probability mass function of the random variables $I$ and $J$ with $n$ MPs

$\bar{I}$ The mean of $I$

$\bar{J}$ The mean of $J$

$T_{s}$ The duration of each successful MMDA four-way handshaking contention

$T_{c}$ The duration of each collision

$T_{\text {MDASRQ }}$ The duration of MDA setup request

$T_{\text {MDASRP }}$ The duration of MDA setup reply

$T_{\text {MDAACK }}$ The duration of MDA acknowledge

$T_{\text {MDAADV }}$ The duration of MDA advertisement

$T_{\text {DIFS }}$ The duration of DIFS

$T_{\text {SIFS }}$ The duration of SIFS

$T_{r}$ The average time to reserve an MDAOP for an MP in $\mathrm{CP}$

$T_{\text {DTIM }}$ The duration of mesh DTIM interval

$T_{\mathrm{DTP}}$ The duration of data transmission period

$T_{i}$ A slot time that should be long enough to access the channel state information

$N_{a}$ The available MDAOPs provided for MPs to reserve in DTP

$N_{c}$ The number of channels in DTP

$B$ The bandwidth of each channel

$D$ MDAOP duration

$L$ The average interval between two MDAOPs

$N_{\min }$ The minimum value of $n$ and $N_{a}$

$T_{m}$ The $m$ th subcritical duration of CP that $m$ MPs can successfully reserve MDAOPs

$X$ The original $\mathrm{CP}$ ratio

$Y$ The shortened $\mathrm{CP}$ ratio

$G$ The number of MPs that can successfully reserve MDAOPs

$S_{\mathrm{NCP}}$ The average throughput of the NCP MP

$T_{\text {cp_s }}$ The duration of shortened CP

$T_{\text {ap }}$ The duration of the additional period

$G_{s}$ The number of MPs that can successfully reserve

MDAOPs in an additional period

$S_{\text {ECPS }}$ The average throughput of the ECPS MP

$\mathrm{Pkt}_{i}$ The total transmitted packets of $\mathrm{MP}_{i}$

$s$ Packet size

$T$ The total simulation time

Tx_Pkt The total successful transmitted packets

$\mathrm{Tl}_{i}$ The time that the $i$ th packet leaves the queue

$\mathrm{Ta}_{i}$ The time that the $i$ th packet enters the queue
Pkt $_{g}$ The numbers of generated packets

$\mathrm{Pkt}_{d}$ The numbers of dropped packets

$S_{i}$ The throughput of $\mathrm{MP}_{i}$

$N_{\text {coll }, i}$ The total collision numbers of $\mathrm{MP}_{i}$

$N_{\text {cont }, i}$ The total contention numbers of $\mathrm{MP}_{i}$

\section{Abbreviations}

ABP: adaptive backoff process; AC: access category; ACK: acknowledge; ADV: advertisement; AIFSN: arbitration interframe space number; AP: access point; BEB: binary exponential backoff; CA: channel assignment;

CBR: constant bit rate; CLFRF: channel load first random fit; CP: contention period; CPC: contention period change; CSMA/CA: carrier sense multiple access with collision avoidance; CW: contention window; DACP: dynamic adjustable contention period; DCF: distributed coordination function; DIFS: DCF interframe space; DP: demarcation point; DTIM: delivery traffic indication message; DTP: data transmission period; ECPS: enable contention period shorten; EDCA: enhanced distributed channel access; ID: identification; LAN: local area network; MAC: medium access control; MAP: mesh access point; MCBF: multichannel best fit; MDA: mesh deterministic access; MDAOP: MDA opportunity; MIFS: MDA interframe space; MMDA: multichannel MDA; MP: mesh point; MPP: mesh point portal; NCP: normal contention period; NMST: neighbor MP status table; QoS: quality of service; SIFS: short interframe space; SMA: scheduled mesh access; SRB: semi-random backoff; STA: station; TRASS: traffic-aware switching scheme; TXOP: transmission opportunity.

\section{Competing interests}

The authors declare that they have no competing interests.

\section{Acknowledgements}

This study was supported in part by the Ministry of Science and Technology (MOST) of Taiwan under Grant No. NSC 99-2221-E-011-119.

\section{Author details}

${ }^{1}$ National Taiwan University of Science and Technology, 43, Section 4, Keelung Rd., Taipei 106, Taiwan. ${ }^{2}$ Ling Tung University, 1, Ling Tung Rd., Taichung 408, Taiwan.

Received: 30 May 2014 Accepted: 26 August 2014

Published: 13 September 2014

\section{References}

1. IEEE, Part 11, Wireless LAN medium access control (MAC) and physical layer (PHY) specifications amendment 10: mesh networking, in IEEE Std. IEEE Std 802, 11s. Draft 2.02, 2008

2. IEEE 802.11 Working Group, Wireless LAN medium access control (MAC) and physical layer (PHY) specifications, 1997

3. YD Lin, SL Tsao, SL Chang, SY Cheng, CY Ku, Design issues and experimental studies of wireless LAN mesh (IEEE Wireless Communications, Apr, 2010). pp. 32-40

4. SM Faccin, C Wijting, J Kenckt, A Damle, Mesh WLAN networks: concept and system design (IEEE Wireless Communications, Apr, 2006). pp. 10-17

5. GR Hiertz, S Max, Y Zang, T Junge, D Denteneer, IEEE 802.11s MAC fundamentals, in Proc. of Mobile Ad-hoc and Sensor Systems, Pisa, 2007, pp. 1-8

6. RC Carrano, LCS Magalhães, DCM Saade, CVN Albuquerque, IEEE802.11s multihop MAC: a tutorial. IEEE Commun Surveys Tutorials 13(1), 52-67 (2011)

7. IEEE std. 802.11e - 2005, Wireless LAN medium access control (MAC) and physical layer (PHY) specifications amendment 8: medium access control (MAC) quality of service enhancements, 2005

8. H Aoki, S Takeda, K Yagyu, A Yamada, IEEE802.11s wireless LAN mesh network technology. NTT DoCoMo Technical J 8(2), 13-21 (2006)

9. M Houda, Z Faouzi, Congestion control in wireless heterogeneous networks, in Proc. of 8th International Conference on Informatics and Systems (INFOS) (Cairo, 2012), pp. 81-85

10. GR Hiertz, S Max, Z Rui, D Denteneer, L Berlemann, Principles of IEEE $802.11 \mathrm{~s}$, in Proc. of 16th International Conference on Computer Communications and Networks, ICCCN 2007, Honolulu, 2007, pp. 1002-1007

11. J Camp, E Knightly, The IEEE802.11s extended service set mesh networking standard. IEEE Commun Mag 46(8), 120-126 (2008)

12. MJ Lee, J Zheng, Y-B Ko, DM Shrestha, Emerging standards for wireless mesh technology, IEEE Wireless Communications, 2006, pp. 56-63 
13. R MacKenzie, T O'Farrell, Achieving service differentiation in IEEE 802.11e enhanced distributed channel access systems. IET Commun 6(7), 740-750 (2012)

14. YW Ahn, J Baek, AMK Cheng, PS Fisher, M Jo, A fair transmission opportunity by detecting and punishing the malicious wireless stations in IEEE 802.11e EDCA network. IEEE Syst J 5(4), 486-494 (2011)

15. P Koutsakis, Token and self-policing-based scheduling for multimedia traffic transmission over WLANs. IEEE Trans Veh Technol 60(9), 4520-4527 (2011)

16. GR Hiertz, S Max, T Junge, D Denteneert, L Berlemann, IEEE 802.11s - Mesh deterministic access, in Proc. of 14th European Wireless Conference, EW 2008, Prague, 2008, pp. 1-8

17. Y He, I Sun, X Ma, AV Vasilakos, R Yuan, W Gong, Semi-random backoff: towards resource reservation for channel access in wireless LANs. IEEE/ACM Trans Networking 21(1), 204-217 (2013)

18. H Cheng, N Xiong, AV Vasilakos, LT Yang, G Chen, X Zhuang, Nodes organization for channel assignment with topology preservation in multi-radio wireless mesh networks. Ad Hoc Netw 10(5), 760-773 (2012)

19. Y Xiao, M Peng, J Gibson, GG Xie, D-Z Du, AV Vasilakos, Tight performance bounds of multihop fair access for MAC protocols in wireless sensor networks and underwater sensor networks. IEEE Trans Mob Comput 11(10), 1538-1554 (2012)

20. V Vishnevsky, A Lyakhov, A Safonov, S Shpilev, Beaconing for MDA support in IEEE 802.11s mesh networks, in Proc. of IEEE 18th International Symposium on Indoor and Mobile Radio Communications (PIMRC 2007), Athens, 2007, pp. 1-5

21. Y Chen, S Emeott, Impact of scheduled mesh access on the capacity of wireless mesh links, in Proc. of Radio and Wireless Symposium, RWS'09, San Diego, 2009, pp. 107-110

22. MDS Islam, MM Alam, CS Hong, JS Sung, Enhanced channel access mechanism for IEEE $802.11 \mathrm{~s}$ mesh deterministic access, in Proc. of Wireless Communications and Networking Conference (WCNC), Sydney, 2010, pp. 1-6

23. A Baiocchi, A Todini, A Valletta, Why a multichannel protocol can boost IEEE 802.11 performance, in Proc. of the 7th ACM International Symposium on Modeling, Analysis and Simulation of Wireless and Mobile Systems, Venice, 2004, pp. 143-148

24. MA Marsan, D Roffinella, Multichannel local area network protocols. IEEE J Selected Areas in Commun 1(5), 885-897 (1983)

25. MA Nezhad, B Bellalta, M Guerrero Zapata, L Cerda Alabern, Should next generation wireless mesh networks consider dynamic channel access? in Proc. of 2nd Baltic Congress on Future Internet Communications (BCFIC), Vilnius, 2012, pp. 32-39

26. JH Lam, SG Lee, WK Tan, Multi-channel wireless mesh networks test-bed with embedded systems, in Proc. of 26th International Conference on Advanced Information Networking and Applications Workshops (WAINA), Fukuoka, 2012, pp. 533-537

27. C-Y Ku, Y-D Lin, S-L Tsao, Y-C Lai, Utilizing multiple channels with fewer radios in wireless mesh networks. IEEE Trans Veh Technol 60(1), 263-275 (2011)

28. BH Lee, HC Chien, HK Wu, Study on multi-channel deterministic access for wireless mesh LAN, in Proc. of the 2012 International Conference on Cyber Technology in Automation, Control, and Intelligent Systems (IEEE-CYBER 2012), Bangkok, 2012, pp. 27-31

29. BH Lee, HC Chien, JM Chen, Study on multi-channel mesh deterministic access for wireless mesh LAN, EURASIP J Wirel Commun Netw, 2012

30. JH Chiang, T Chiueh, Accurate clock synchronization for IEEE 802.11-based multi-hop wireless networks, in Proc. of 17th IEEE International Conference on Network Protocols (ICNP 2009), Princeton, 2009, pp. 11-20

31. H Jasani, N Alaraje, Evaluating the performance of IEEE 802.11 network using RTS/CTS mechanism, in Proc. of IEE International Conference on Electro/ Information Technology (EIT 2007), Chicago, 2007, pp. 616-621

32. J Hoblos, Fairness Enhancement in IEEE 802.11s Multi-hop wireless mesh networks, in Proc. of IEEE 13th International Conference on Communication Technology (ICCT), Jinan, 2011, pp. 647-651

33. BJ Kwak, NO Song, LE Miller, Performance analysis of exponential backoff. IEEE/ACM Trans Networking 13(2), 343-355 (2005)

doi:10.1186/1687-1499-2014-149

Cite this article as: Chien et al:: A dynamic adjustable contention period mechanism and adaptive backoff process to improve the performance for multichannel mesh deterministic access in wireless mesh LAN. EURASIP Journal on Wireless Communications and Networking 2014 2014:149.

\section{Submit your manuscript to a SpringerOpen ${ }^{\odot}$ journal and benefit from:}

- Convenient online submission

- Rigorous peer review

- Immediate publication on acceptance

- Open access: articles freely available online

- High visibility within the field

- Retaining the copyright to your article

Submit your next manuscript at $\gg$ springeropen.com 